\title{
Chronic Obstructive Pulmonary Disease (COPD) Screening is Still a Challenge in Pakistan
}

\author{
Anam Rehman, Hamid Shafiq, Shireen Jawed, Fakiha Behram
}

\begin{abstract}
Background:Chronic obstructive lung disease (COPD) is a highly preventable disease with smoking as most recognizable risk factor globally. COPD remains undiagnosed worldwide due to unavailability of gold standard spirometry. Technical difficulties in this procedure, faced by patient result in over and under diagnosis of this prevalent disease. Objective of this study was to screen the undiagnosed air way obstruction which reflects the COPD among the smokers and nonsmokers. PEFR was used for assessment, as it is simple, reliable and easily available way at most of the health care centers.

Methodology:A cross sectional study was conducted from May 2018 to Dec 2018 at private medical institute and private hospital in Faisalabad. It comprised of 200 males (smokers and nonsmokers) who had no previous history of diagnosis of COPD. Relevant information related to smoking including pack years and medical history specially related to COPD was inquired on structured questionnaire. Air way obstruction was assessed by PEFR using vitalograph compact. Data was analyzed by SPSS 21 version. Continuous variables are presented as mean and standard deviation (SD) and compared by Independent t-test among study groups. Categorical variables are expressed as proportions and compared by $\mathrm{X}^{2}$ test. $\mathrm{P}$ value $\leq 0.05$ was considered significant.

Results: Results are showing that $91.56 \%$ of the studied smokers have air way obstruction based on yellow and red zones of PEFR. Out of total COPD smokers $62.7 \%$ were in yellow zone and $28.9 \%$ were in red zone. Only $29.1 \%$ of nonsmokers have PEFR values within yellow. None of nonsmoker was in red zone (P value $0.001 *)$. PEFR was significantly lower in smokers in comparison to nonsmokers (P value $0.003 *) .95 .5 \%$ of Subjects in red zone were symptomatic, while only $51.2 \%$ of subjects in yellow zone were symptomatic considering mild to severe air flow obstruction.
\end{abstract}

Conclusion:Smokers and nonsmokers have undiagnosed airway obstruction with symptoms reflecting COPD but greater proportion of smoker were involved. PEFR was lower in smokers as compared to nonsmokers.

Key Words: Air way obstruction, undiagnosed COPD, smokers, nonsmokers, PEFR, spirometry

\section{Introduction}

Chronic obstructive pulmonary disease (COPD) is a progressive pulmonary disorder associated with poor quality of life and one of the leading cause of mortality worldwide. ${ }^{1}$ It is a global public health crisis with smoking being recognized as its most important causative factor. ${ }^{2,3}$ It is characterized by persistent respiratory symptoms and air flow limitation due to air way or alveolar abnormalities caused by exposure to toxic chemicals or gases. ${ }^{1}$ Global prevalence of COPD is $11.7 \%$ with 3 million death annually. ${ }^{1,4}$ It is currently $4^{\text {th }}$ leading cause of mortality across the globe which is expected to be $3^{\text {rd }}$ leading cause of death by the year

\section{Dr. Anam Rehman}

Senior Demonstrator

Aziz Fatimah Medical and Dental College, Faisalabad.

Maj.Gen. (R) Prof. Dr. Hamid Shafiq HI(M)

Professor

Aziz Fatimah Medical and Dental College, Faisalabad.

Dr. Shireen Jawed,

Associate Professor

Aziz Fatimah Medical and Dental College, Faisalabad.

Fakiha Behram

Final year Student (BS BioTech)

Foreman Christian College LHR (FC College)

Correspondence:

Dr. Shireen Jawed

Email: drshireenjawed@gmail.com
2020. ${ }^{1}$ Existing prevalence data related to COPD varies universally due to different surveys techniques, diagnostic criteria and approaches. ${ }^{1}$ Burden of this disease is continuously increasing at alarming rate because of continued exposure to its risk factor specially smoking. Due to increasing prevalence of smoking in developing countries, burden of COPD is expected to rise by next 30 years and by 2030 death rates are expected to be increased by 4.5 million due to COPD and related conditions. ${ }^{1}$ The countries where tobacco consumption is higher are at risk of increasing burden of this disease. Early stages of the COPD can be asymptomatic which can be prevented to progress by smoking cessation, increasing physical activity, and early detection and treatment. ${ }^{5,4}$ Lower estimates of prevalence worldwide are based on self-reporting of a physician's diagnosed COPD, only a small proportion have documented spirometry findings across the world. ${ }^{1.2}$ This reflects the under or over diagnosis of COPD. ${ }^{1}$ It remains undiagnosed in many subjects with persistent airflow limitation particularly in developing countries with poor resources. ${ }^{2,6}$ Undiagnosed individuals may be susceptible to exacerbation-like pneumonia, pneumothorax, and premature mortalities due to missed opportunities for 
prevention, modification of risk factors and observation of lung function decline particularly among the smokers. ${ }^{6}$ Previous epidemiological studies reported that $75 \%$ of COPD patients remain ${ }^{5,7}$ undiagnosed due to scarcity of clinical suspicion and under use of spirometry as it is not widely available. ${ }^{8,9}$ Gold standard test for diagnosis of COPD is spirometry but technical difficulties in performing this procedure and unavailability of spirometer and trained technicians frequently limit usage, especially at basic health units (BHU); providing primary health care level universally. ${ }^{7,10}$ Although this procedure is a non-invasive and safe and reproducible but its use is not feasible at many clinical setups. In the private setups this procedure is expensive and an unaffordable test for many patients in resource-poor countries. ${ }^{8,9}$ Forced expiratory volume in 1 second (FEV1) and force vital capacity (FVC) are spirometric parameter used for assessment of lung function and diagnosis of symptomatic COPD. Early stages of COPD can be asymptomatic and frequently be misdiagnosed until it is clinically apparent and advanced, ${ }^{9}$ therefore spirometry is not recommended for these individuals. ${ }^{1}$ Spirometric measurements are not always valid because most of the patients have difficulties in expiring fully in order to provide FVC and remain undiagnosed. ${ }^{7,10}$ According to Global Initiative for Chronic Obstructive Lung Disease (Gold) report that FEV1 is unreliable marker to assess the severity of breathlessness, exercise limitation and health status impairment. ${ }^{1}$ In this context, PEFR measurement is widely available alternate to spirometry. ${ }^{8}$ It is expiratory parameter obtained by peek flow meter or compact vitalograph. It is a simple index of pulmonary functions which measures the caliber of the airways and commonly used in researches and clinical practices. ${ }^{11}$ The main advantages of PEFR are simplicity and reliability of its procedure. ${ }^{10}$ It is a valuable tool in lung functions studies for diagnosis, assessment of severity of airway obstruction and selection of the treatment regimes. ${ }^{10,11}$ Various clinicians can develop COPD management plans including admissions and discharges of patients based on green-yellow-red zones of PEFR classification as per guidelines by American lung association due to availability of peak flow meter in clinical setting. ${ }^{11}$ It is highly sensitive and accurate index of airway obstruction. Air way obstruction in smokers is symptomless in earlier stages, ${ }^{12}$ but can be easily picked by PEFR. ${ }^{13}$ PEFR may not be able to accurately identify GOLD stage, but previous studies reported usefulness of peak flow rate measurements in COPD screening following subsequent confirmatory spirometry. ${ }^{9,10}$ Under reporting of the symptoms by the patients and poor assessment by the primary health care professionals due to lack of feasibilities including spirometry may results in high prevalence of this public health crisis. ${ }^{12}$ Initiatives for early detection and treatment of COPD and cessation of cigarette smoking prevent the loss of lung functions and produce Public health benefits. Primary health care professionals may play significant role in early and accurate diagnosis of COPD by screening all the patients in high risk group like chronic smokers by using PEFR as alternative to spirometry during their primary care visits. Global Initiative for Chronic Obstructive Lung Disease (GOLD) encourages new researches worldwide in this highly prevalent disease to develop innovative approaches for early screening of undiagnosed COPD and planning its management and prevention to reduce its burden on population. ${ }^{1}$ According to GOLD report revised in 2018, no data is available showing the effectiveness of spirometry in the diagnosis, management and improving COPD outcomes in the individuals without developing significant symptoms. ${ }^{1}$ GOLD criteria recommend spirometry for only symptomatic subjects but not for screening asymptomatic subjects. Keeping this in mind this study was designed to screen undiagnosed air way obstruction which reflects COPD patients among smokers and nonsmokers using PEFR as a diagnostic tool.This procedure is simple, reliable and easily available, therefore it is proposed as a surrogate of spirometry for diagnosis of COPD.

\section{Objectives}

To investigate the undiagnosed air way obstruction which reflect the COPD among smokers and nonsmokers subjects using PEFR as a diagnostic tool.

To compare PEFR among smokers and nonsmokers.

\section{Methodology}

This cross sectional study was conducted at private medical college and hospital in Faisalabad from May 2018 to Dec 2018. It comprised of 200 male subjects of 20 to 40 years age. After taking ethical approval from the ethical committee of institute and hospital, participants were selected from the employees of above mention centers by non-probability convenience sampling technique. Initially 250 subjects were approached and relevant information about ethnicity, occupation, and lifestyle, history of smoking including duration and pack years (number of cigarettes per day by years), history of persistent dyspnea, cough, sputum, chest pain, allergies and frequent respiratory infections, systemic and respiratory diseases were recorded on structured proforma. Drug history specially related to air way obstruction like bronchodilators and steroids was 
taken. Questions related to assessment of breathlessness and cough were also emphasized as these are two main symptoms along with air flow limitation recommended for diagnosis of COPD (according to Gold criteria). ${ }^{1}$ Selfreported undiagnosed male cigarette smokers of age 2040years (who reported smoking more than 100 cigarettes in their life regularly ${ }^{12}$ and had no history of previous diagnosis of COPD and treatment for airway obstruction) and nonsmokers (who never smoke or smoke less than 100 cigarettes in their life and don't have previous history of COPD or treatment for airway obstruction) of the same age were included in the study. Female smokers to prevent the gender bias, subjects with cigar, shisha and other types of smoking, subjects who were unable to perform procedure and with thoracicspine deformities (kyphosis, scoliosis and fused ribs) were excluded. Subjects who had previous diagnosis of respiratory diseases including, asthma/COPD, restrictive lung disease, tuberculosis and lung cancers and with history of systemic diseases affecting the respiratory system, subject on existing or previous therapies like bronchodilators and steroids for airway obstruction were also excluded from this study. After taking informed consent the anthropometric measurements including height in centimeters and weight in $\mathrm{kg}$ were obtained by stadiometer. BMI was calculated by weight in $\mathrm{kg} /$ height in $\mathrm{m}^{2}$ and preliminary clinical examination was done to exclude any systemic disorder affecting respiratory system. PEFR was recorded by calibrated vitalograph compact following standard guidelines by American Thoracic Society (ATS). The subjects were instructed to take deep breath and exhale with forced expiration as hard as possible in a single blow into the apparatus while maintaining an air tight seal between lip and mouth piece. To avoid contamination disposable mouthpiece was used for each subject. Test manoeuvre for each patient was repeated three times and highest reading was considered for analysis. PEFR readings were classified into three zones as per American Lung Association. PEFR of 80 to $100 \%$ was considered as green zone (safe zone), while between 50 to $80 \%$ was classified as yellow zone (alert zone) and $<50 \%$ was red zone (zone of emergency). ${ }^{11}$

Statistical analysis

Statistical analysis was conducted on SPSS 21 version. Descriptive data including age, height, weight, BMI and PEFR is described as mean \pm standard deviation (SD). Mean values for PEFR among smokers and nonsmokers was compared by independent t-test. Undiagnosed COPD was analyzed as a dichotomous outcome (absence versus presence) and presented as frequencies and percentages. Proportions of subjects with green-yellowred zone of PEFR classification for air way obstruction were reported. Chi square test $\left(\mathrm{X}^{2}\right)$ was used to compare the proportions of COPD among smokers and nonsmoker. Percentages of symptoms were also compared by $\mathrm{X}^{2}$ test. $\mathrm{P} \leq 0.05$ was taken as statistically significant.

\section{Results}

Study population comprised of 200 male subjects with mean age $21 \pm 4.72$. Basic characteristics of study population are presented in table 1 . Table 2 is showing the frequencies and percentages of COPD among smokers and nonsmokers based on PEFR classification (greenyellow and red zone). Results reveal that $91.56 \%$ of the studied smokers had air way obstruction based on yellow and red zones of PEFR. Out of total COPD smokers $62.7 \%$ of the smokers were in yellow zone and $28.9 \%$ were in red zone considering mild to severe air flow obstruction. In contrast to smokers, only $29.1 \%$ of nonsmokers had PEFR values in yellow zone, being in early stages of air flow obstruction. None of the nonsmoker was in red zone. $\mathrm{X}^{2}$ test shows significant difference among both groups (p value $0.001^{*}$ ) (Table 2 ). Subjects in yellow zone (alert zone) and red zone (emergency zone) reported cough with sputum, breathlessness, wheeze and chest tightness but they did not take any medication for airway obstruction nor they were screened for COPD before enrollment in study. (self-reported) (Table 3). 95.5\% of Subjects in red zone were symptomatic, while only $51.2 \%$ of subjects in yellow zone, showing early stages of air flow obstruction, were symptomatic. Rest were asymptomatic. Based on PEFR zones and presence of symptoms, current study found that $108(54 \%)$ of total population including smokers and nonsmokers have undiagnosed COPD.

Table 1: Descriptive of studied subjects $(\mathrm{N}=\mathbf{2 0 0})$

\begin{tabular}{|c|c|}
\hline Anthropometric Measurements & Mean \pm SD \\
\hline Age $(\mathrm{age})$ & $21.89 \pm 4.72$ \\
\hline Height $(\mathrm{cm})$ & $158.38 \pm 13.09$ \\
\hline Weight $(\mathrm{kg})$ & $62.04 \pm 12.60$ \\
\hline BMI $(\mathrm{kg} / \mathrm{m} 2)$ & $24.62 \pm 3.42$ \\
\hline PEFR $(\mathrm{L} / \mathrm{S})$ & $5.88 \pm 1.03$ \\
\hline
\end{tabular}

BMI= Body mass index, PEFR ( Peak expiratory flow rate)

$\mathrm{SD}=$ Standard. Deviation, $\mathrm{cm}=$ centimeters, $\mathrm{m}=$ meters

$\mathrm{Kg}$ (kilogram), L /S (liters/ seconds)

(Table 3) Figure1 is showing the comparison of mean PEFR by independent $t$-test. It is indicating that PEFR is significantly lower in smokers in contrast to nonsmokers (P value $0.003^{*}$ ). Regression analysis is showing the significant positive association of PEFR with height 
( $\beta=0.005, \mathrm{P}$ value $\left.0.030^{*}\right)$.PEFR is not significantly associated with age $(\mathrm{P}$ value $=0.12), \mathrm{BMI}(\mathrm{P}$ value 0.41$)$ and weight (P value 0.34) (Table 3 )

Table 2: Frequencies and Percentages of COPD among the Study Population Based on PEFR Values $(\mathrm{N}=\mathbf{2 0 0})$

\begin{tabular}{|l|c|c|c|c|c|}
\hline \multirow{4}{*}{$\begin{array}{c}\text { Study } \\
\text { Groups }\end{array}$} & $\begin{array}{c}\text { Subject without } \\
\text { COPD } \\
\text { N (\%) }\end{array}$ & \multicolumn{3}{|c|}{$\begin{array}{c}\text { Subjects with COPD } \\
\text { N (\%) }\end{array}$} & \multirow{2}{*}{$\begin{array}{c}\text { Total } \\
\text { ( 200) }\end{array}$} \\
\cline { 2 - 5 } & $\begin{array}{c}\text { Green Zone } \\
\text { PEFR } \\
(\mathbf{1 0 0 - 8 0 \% )}\end{array}$ & $\begin{array}{c}\text { Yellow Zone } \\
\text { PEFR } \\
\text { (80-50\%) }\end{array}$ & $\begin{array}{c}\text { Red Zone } \\
\text { PEFR } \\
(<\mathbf{5 0 \% )}\end{array}$ & Total & \\
\hline Smokers & $7(8.4)$ & $52(62.7)$ & $24(28.9)$ & $76(91.56)$ & 83 \\
\hline $\begin{array}{l}\text { Non } \\
\text { Smokers }\end{array}$ & $83(70.9)$ & $34(29.1)$ & 00 & $34(29.1)$ & 117 \\
\hline P Value & & \multicolumn{3}{|c|}{$0.001 *$} & \\
\hline
\end{tabular}

Percentages are compared by $\mathrm{X}^{2}$ test,

Statistically significant at $\mathrm{P}$ value $\leq 0.05$

Table 3: Pulmonary Symptoms Based on PEFR Zones ( $\mathrm{N}=108)$

\begin{tabular}{|l|c|c|}
\hline \multirow{4}{*}{ Symptoms } & \multicolumn{2}{|c|}{$\begin{array}{c}\text { Subjects with COPD } \\
\text { N= 108 }\end{array}$} \\
\cline { 2 - 3 } & $\begin{array}{c}\text { Yellow Zone (n=86) } \\
\text { PEFR (80 -50\%) }\end{array}$ & $\begin{array}{c}\text { Red Zone(n=22) } \\
\text { PEFR (<50\%) }\end{array}$ \\
\cline { 2 - 3 } & N(\%) & N(\%) \\
\hline Productive cough & $11(12.8)$ & $17(70.8)$ \\
\hline Dry cough & $6(6.97)$ & $8(36.3)$ \\
\hline Breathlessness & $30(34.9)$ & $15(62.5)$ \\
\hline Wheeze & $3(3.5)$ & $10(45.5)$ \\
\hline Chest tightness & $18(20.9)$ & $13(59.5)$ \\
\hline Symptomatic & $44(51.2)$ & $1(4.5)$ \\
\hline Asymptomatic & $42(48.8)$ & $0.004 *$ \\
\hline P value & \multicolumn{2}{|c}{} \\
\hline
\end{tabular}

Symptoms are self-reported by study participants $\mathrm{X}^{2}$ test was used to check difference in percentages Statistically significant at $\mathrm{P}$ value $\leq 0.05$.

Figure1: Comparison of PEFR among study groups $(\mathrm{N}=\mathbf{2 0 0})$

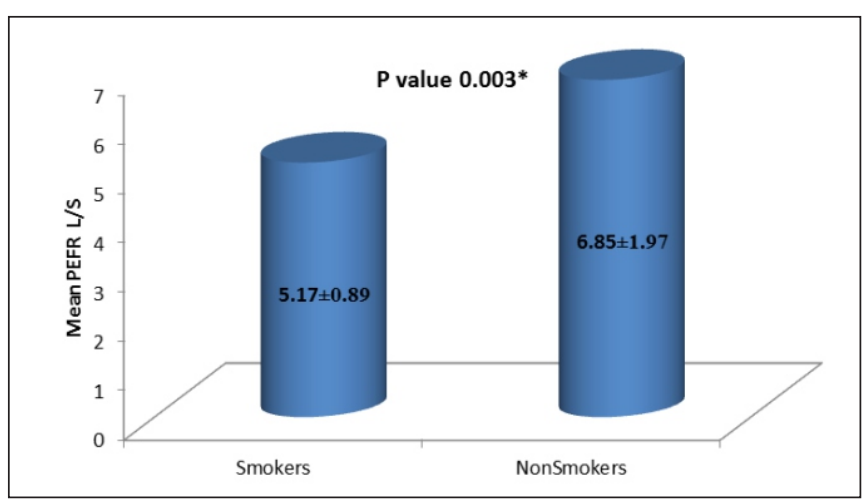

PEFR $=$ Peak expiratory flow rate, comparison by t-test Statistically significant at $\mathrm{P}$ value $\leq 0.05$

\section{Discussion}

Current study was conducted to highlight the chronic pulmonary obstructive disease (COPD) which is highly preventable but manageable disease. Air flow limitation is the hallmark of this disease which results from continuous exposure to noxious particles or gases. ${ }^{1}$ Cigarette smoking is the most common risk factor for
COPD in developing countries adversely affecting the lung functions. ${ }^{14}$ Most of the COPD patients remain undiagnosed worldwide due to unavailability of gold standard procedure, spirometry in routine clinical practices specially at primary health care centers. ${ }^{8,15} \mathrm{It}$ is expensive and unaffordable for many middle and lower class patients. Most of the patients fail to perform this procedure accurately, resulting in under or over diagnosis of COPD. Previous researches reported that only small proportion of physician-diagnosed COPD patients have documented spirometry findings available globally. ${ }^{12}$ Due to lack of structured COPD screening program, and uncommon use of spirometry for the evaluation of patients with chronic respiratory symptoms, the prevalence of undiagnosed air way obstruction is anticipated to be high, especially among chronic smokers who are high-risk population for this ailment. ${ }^{12}$ In comparison to this PEFR measurements are easy and widely available. It is an important mean to assess the lung functions and airway obstruction and has pivotal role in diagnosis and prognosis of obstructive and hyperactive pulmonary diseases. ${ }^{16}$ It can be easily used in screening the population at risk of COPD as it is inexpensive and affordable. In contrast to spirometry, it is a simple procedure and patient can easily perform it. Early screening of obstructive disease with PEFR and cessation of cigarette smoking can prevent its damaging effects on respiratory system. ${ }^{16}$ Current study found significant reduction in PEFR in smokers than nonsmokers (5.17 Vs 6.85). This finding was consistent with Sawant GV et al, Mukherjee $\mathrm{S}$ and various other studies who also documented decline in pulmonary function and PEFR $^{16,17}$ Inflammation is the hallmark of cigarette smoking. Hyper-secretion of inflammatory mediators results in hyper-reactivity and increase in tone of alveolar smooth muscles. ${ }^{16}$ Broncho-pulmonary leakage and edema further contribute to alveolar walls thickness and airway narrowing affecting both small and large airways. ${ }^{17,19}$ Collectively all these factors promote airway obstruction limiting air flow, decrease diffusing capacity and subsequent decline in PEFR. ${ }^{18}$ Quitting smoking can reverse these harmful consequences. ${ }^{8}$ However, persistent tobacco smoking can progresses to permanent destruction of alveolar walls and deforming bronchopulmonary passage worsening the ventilatory function. ${ }^{11}$ Some previous studies also documented reduced diffusing capacity in smokers most probably due to a lower pulmonary capillary blood flow in smokers in contrast to non-smokers. ${ }^{13}$ All these changes are the possible explanation of limited ventilatory functions among smokers. Current study found that $91.5 \%$ of the smokers have undiagnosed airway obstruction based on PEFR. By classifying the severity of air way obstruction using zones guided by American lung association, out of total COPD smokers $62.7 \%$ have PEFR values within 70$50 \%$ and lie in yellow zone (zone of alert) having mild to moderate airway obstruction. $28.9 \%$ COPD Smokers are in red zone (emergency zone) with PEFR values $<50 \%$ 
showing severe COPD.In contrast to smokers only $29.1 \%$ of nonsmokers have PEFR values within yellow zone indicating early stages of COPD. None of them is in red zone.Subjects in yellow and red zone were considered as undiagnosed COPD because they also report on and off cough, sputum and breathlessness but they don't take any medication for air way obstruction. Similar findings were also presented by Sawant GV, who reported lower PEFR readings in $84 \%$ of smokers as compared to $60 \%$ among nonsmokers. ${ }^{16}$ These findings suggest that cigarette smoking is the key factor to influence airway obstruction in smokers and limit ventilatory function. ${ }^{20}$ This study also found that $95.5 \%$ of subjects in red zone are symptomatic, while only $51.2 \%$ of subjects in yellow zone are symptomatic and rest are asymptomatic, indicating early stages of airway obstruction can be asymptomatic. These findings are supported by cohort study conducted in Denmark which reported asymptomatic cases of undiagnosed COPD in general population. ${ }^{5}$ This study adds pertinent information about COPD which is a common hazard of tobacco smoking. Early diagnosis of COPD may motivate smokers for quitting smoking, which is the only measure to improve future prospects of chronic smokers.

Conclusion

PEFR was lower in smokers as compared to nonsmokers. Smokers and nonsmokers have undiagnosed airway obstruction with symptoms reflecting COPD but greater proportions of smokers were involved showing mild to severe condition. PEFR can be used as a simple tool to screen population being at high risk.

Limitation and Recommendations

I. PEFR screening of high risk population such as smokers should be done in order to diagnose COPD at early stages with an emphasis for cessation of smoking.

II. Health awareness programs should be increased to motivate people to quit smoking in order to prevent morbidity and mortality associated with it.

$\begin{array}{ll}\text { Funding Source: } & \text { Nil } \\ \text { Conflicts of interest: } & \text { Nil }\end{array}$

\section{Reference}

1. Global Initiative for Chronic Obstructive Lung Disease (GOLD). Global strategy for the diagnosis, management, and Prevention of chronic obstructive lung diseases (GOLD). 2018report. https://goldcopd.org/wpcontent/uploads/2017/11/GOLD-2018-v6.0-FINALrevised-20-Nov_WMS.pdf

2. Labonté LE, Tan WC,Li Pz,et al. Undiagnosed chronic obstructive pulmonary disease contributes to the burden of health care use. Data from the Can Cold study. Am J Respir Crit Care Med. 2016; 194: 285-298
3. Laniado-Laborín R. Smoking and chronic obstructive pulmonary disease (COPD). Parallel epidemics of the 21st century. Int J Environ Res Public Health. 2009 ;6(1):209-24.

4. Rabe KF, etal chronic obstructive pulmonary disease. Lancet.2017;389 (100082):1931-1940.

5. Çolak Y, Afzal S, Nordestgaard BG, Vestbo J, Lange P Prognosis of asymptomatic and symptomatic, undiagnosed COPD in the general population in Denmark: a prospective cohort study. Lancet Respir Med. $2017 ; 5(5): 426-434$.

6. Martinez CH Mannino DM Jaimes FA etal. Undiagnosed obstructive lung disease in the United States. Associated factors and long-term mortality. Ann Am Thorac Soc. 2015; 12: 1788-1795.

7. Almagro P, Soriano JB. Underdiagnosis in COPD: a battle worth fighting. The Lancet Respiratory Medicine. 2017;5(5):367-368.

8. Pothirat C, Chaiwong W, Phetsuk N, Liwsrisakun C, Bumroongkit C, Deesomchok A, Theerakittikul T, Limsukon A. Peak expiratory flow rate as a surrogate for forced expiratory volume in 1 second in COPD severity classification in ThailandInt J Chron Obstruct Pulmon Dis. $2015 ; 10$ : 1213-1218.

9. Fir Perez-Padilla R, Vollmer WM, Vzquez-Garcia JC, et al. Can a normal peak expiratory flow exclude severe chronic obstructive pulmonary disease? Int J Tuberc Lung Dis. 2009;13(3):387-393.

10. Mahboub B, Alzaabi A, Soriano JB, et al. Casefinding of chronic obstructive pulmonary disease with questionnaire, peak flow measurements and spirometry: a cross-sectional study. BMC Res Notes. 2014 ; 7:241. doi: 10.1186/1756-0500-7-241.

11. Nighute S, Buge K, Kumar S. Effect of cigarette smoking on peak expiratory flow rate: a short review. Int. J. Curr. Res. Physiol. Pharmacol. 2017; 1(1):3-5. 
12.Fu SN, Yu WC, Wong CK, Lam MC.Prevalence ofundiagnosedairflow obstruction among people with a history of smoking in a primary care setting. Int J Chron Obstruct Pulmon Dis. 2016 ;11:2391-2399. E Collection 2016.

13. Mridha MAA, Amin MR, Kabir ARML, Peak Expiratory Flow Rate (PEFR)-A Simple Ventilatory Lung Function Test J ShaheedSuhrawardy Med Coll, 2011;3(2): 44-47.

14. Medabala T, Rao BN, Glad Mohesh MI, Kumar P. Effect of cigarette and cigar smoking on peak expiratory flow rate. Journal of clinical and diagnostic research: JCDR. 2013;7(9):1886.

15. Stafyla E, Kotsiou OS, Deskata K, Gourgoulianis KI Missed diagnosis and over treatment of COPD among smoking primary care population in Central Greece:old problems persist. Int J Chron Obstruct Pulmon Dis.2018;5:13:487-498.

16. Sawant GV, Kubde SR, Kokiwar PR. Effect of smoking on PEFR: a comparative study among smokers and non smokers in an urban slum community of Hyderabad, India. International Journal Of Community Medicine And Public Health. 2017;3(1):246-50.

17. Mukherjee S, Banerjee G, Mahapatra ABS. Peak Expiratory Flow Rate Changes with Relevant Variables in a Population of Eastern India. Indian J Physiol Pharmacol 2018; 62(3) :372-379.

18. Kaur H, Singh J, Makkar M, Singh K, Garg R. Variations in the peak expiratory flow rate with various factors in a population of healthy women of the malwa region of Punjab, India. Journal of clinical and diagnostic research: JCDR. 2013; 7(6):1000.

19. Bajentri AL, Veeranna N, Dixit PD, Kulkarni SB. Effect of 2-5 years of tobacco smoking on ventilatory function tests. J Indian Med Assoc. 2003; 101(2):96- 108.

20. Medabala T, Rao BN, Mohesh GM, Praveenkumar MP. The effect of ageing on vital capacity and peak expiratory flow rate in healthy non-smoking agricultural workers. Int J Med Health Sci. 2012;1(4):47-52.

\section{Author`s Contribution}

Dr. Anam Rehman: Study design, data collection, Interpretation of results, manuscript writing

Maj. Gen. (R) Prof. Dr. Hamid Shafiq HI (M): Study design, supervise all the research work Reviewed and approved the manuscript.

Dr. Shireen Jawed: Acquisition of data, statistical analysis, interpretation of results, formulation of tables, writing the manuscript. Reviewed and approved the manuscript 